\title{
452351 - QI REVIEW OF THREE EPIDURAL SOLUTIONS FOR POST-OP ANALGESIA
}

\author{
George Evans, MD, Neal Badner, MD, FRCP(C), George Nicolaou, MD, FRCPC, \\ Dave Smith, MD, FRCPC \\ Anesthesiology, University of Western Ontario, London, ON, Canada
}

Introduction: This chart review was a quality improvement project to compare three epidural solutions that have been used at a tertiary care academic hospital. The three solutions compared were (1) 0.1\% Bup./Dilaudid 20ug/cc, (2) 0.125\% Bup./Dilaudid 20ug/cc and (3) 0.125\% Bup./Fentanyl 2ug/cc.

Methods: Local REB approval was obtained for this study. Retrospective Chart Review of Patients that had undergone elective AAA or Thoracic Surgery (Lobectomy, Pneumonectomy) with preoperative placement of an Epidural catheter. Medical Records generated a list from May, 2005 to July, 07'; patients were selected continuously until at least 200 met inclusion criteria. End points included hypotension (SBP $<90 \mathrm{mmHg}$ at least 60 min apart), fluid requirements, mean pain scores, mean infusion rates and side effects.

Results: See Table 1

Discussion: There was only one significant difference in baseline characteristics between groups, which was slightly less ASA 4 patients in Thoracic group (3). There was no difference in significant outcomes including MI, arrhythmia or acute renal failure. The significant differences in infusion rates demonstrated that a higher volume of 0.125 $\mathrm{Bup} / \mathrm{F}$. was required for adequate analgesia. The likely explanation for the significant difference in mean pain scores in the Thoracic group (1) was the more potent Dilaudid over Fentanyl and that in group (2) infusion was held for a longer time period when hypotension occurred. Among AAA patients, pain scores were actually higher with the most potent solution (2), this appears to correlate with the significant difference in this solution being held for hypotension. There was a higher incidence of nausea and vomiting in Thoracic patients recieving solutions with Dilaudid, however this difference was not seen in AAA patients. Many other combinations of local anesthetic and an opioid are possible; several studies have looked at different combinations, their effects on pain control and side effects [1][2][3]. However, few studies have looked at incidence of hypotension. It appears that changing to lower concentration of Bup. along with a less soluble more potent opioid, resulted in better post op pain control and a trend toward less hypotension. This information has lead to a change to solution (1) at this institution. References: [1] C. N. H. Tan et al. (2004) British Journal of Anaesthesia, Vol. 92, No. 5 670-674 [2] PS Hodgson et al (2001) Anesth Analg; 92:1024-1028 [3] A Macias et al (2002) Anesth Analg 2002; 95:1344-1350

Table 1: Results: Significant endpoints 


\begin{tabular}{|c|c|c|c|c|}
\hline AAA & $\begin{array}{l}\text { (1)BUP0.1/D } \\
\text { [N=19] }\end{array}$ & (2)BUP0.125/D $[\mathrm{N}=44]$ & (3)BUP0.125/F[N=31] & $\begin{array}{c}\mathrm{P} \\
\text { VALUE }\end{array}$ \\
\hline $\begin{array}{l}\text { Soln. Rate (cc/hr) } \\
\text { POD } 0\end{array}$ & $6.68(1.00)$ & $6.57(1.81)$ & $7.58(1.69)$ & .028 \\
\hline $\begin{array}{l}\text { Soln. Rate (cc/hr) } \\
\text { POD } 1\end{array}$ & $6.32(1.20)$ & $6.45(2.03)$ & $7.55(2.01)$ & .026 \\
\hline $\begin{array}{l}\text { Epidural held (>1 } \\
\text { min) POD } 0\end{array}$ & $0(0.0 \%)$ & $12(27.3 \%)$ & $8(25.8 \%)$ & .040 \\
\hline $\begin{array}{l}\text { Pain score at rest } \\
\text { POD } 1\end{array}$ & $2.16(1.68)$ & $1.80(1.54)$ & $3.55(2.34)$ & $<.001$ \\
\hline $\begin{array}{l}\text { Pain score with } \\
\text { activity POD } 1\end{array}$ & $4.63(2.13)$ & $4.19(2.03)$ & $6.23(2.61)$ & .003 \\
\hline $\begin{array}{c}\text { VAS }(>7)-\text { Any POD } \\
0\end{array}$ & $3(15.8 \%)$ & $18(41.9 \%)$ & $4(13.3 \%)$ & .012 \\
\hline Thoracics & $\begin{array}{c}\text { (1)Bup } \\
0.1 / \mathrm{D}[\mathrm{n}=32]\end{array}$ & (2)Bup 0.125/D [n=46] & (3)Bup 0.125/F [n=35] & P Value \\
\hline $\begin{array}{c}\text { Total episodes of } \\
\text { hypotension/day in } \\
\text { those } 1+\text { episode POD } \\
1\end{array}$ & $\begin{array}{c}\mathrm{n}=8, \\
\text { mean }=2.75 \text {, } \\
\text { sad }=1.04\end{array}$ & $\begin{array}{c}\mathrm{n}=21, \text { mean }=4.43, \\
\text { sad }=2.44\end{array}$ & $\begin{array}{c}\mathrm{n}=14, \text { mean }=2.36 \text {, } \\
\text { sad }=1.60\end{array}$ & $\mathrm{p}=, 015$ \\
\hline $\begin{array}{l}\text { Soln. Rate (cc/hr) } \\
\text { POD 0 }\end{array}$ & $7.00(1.65)$ & $6.59(1.84)$ & $8.46(2.55)$ & $<.001$ \\
\hline $\begin{array}{l}\text { Soln. Rate (cc/hr) } \\
\text { POD } 1\end{array}$ & $6.47(1.98)$ & $6.41(1.86)$ & $7.86(2.70)$ & .008 \\
\hline $\begin{array}{l}\text { Soln. Rate (cc/hr) } \\
\text { POD } 2\end{array}$ & $6.00(2.44)$ & $6.21(2.03)$ & $7.94(2.85)$ & .002 \\
\hline $\begin{array}{l}\text { Pain score at rest } \\
\text { POD } 0\end{array}$ & $2.63(2.24)$ & $3.11(1.89)$ & $3.91(2.28)$ & .050 \\
\hline $\begin{array}{l}\text { Pain score at rest } \\
\text { POD } 1\end{array}$ & $1.50(1.83)$ & $2.09(1.85)$ & $2.71(2.14)$ & .045 \\
\hline $\begin{array}{l}\text { Pain score with } \\
\text { activity POD } 0\end{array}$ & $2.76(2.54)$ & $4.82(2.53)$ & $4.65(3.41)$ & .015 \\
\hline $\begin{array}{l}\text { N \& Vomiting } \\
\text { Score }>0 \text { POD 0 }\end{array}$ & $5(15.6 \%)$ & $6(13.3 \%)$ & $0(0.0 \%)$ & .032 \\
\hline $\begin{array}{l}\text { Itchiness and } \\
\text { Treatment POD 0 }\end{array}$ & $1(3.1 \%)$ & $8(17.4 \%)$ & $1(2.9 \%)$ & .048 \\
\hline
\end{tabular}

